chronic graft-versus-host disease model and dermal fibrosis in the tightskin-1 model of systemic sclerosis. Effects on the TGF- $\beta / S m a d$ signaling pathway were investigated in vivo. Exposure of AM1476 was measured to ensure proper target engagement.

Methods: The murine sclerodermatous chronic graft-versus-host disease (cGvHD) model was used to evaluate anti-fibrotic effects after therapeutic dosing of the $5-\mathrm{HT}_{2 \mathrm{~B}}$ receptor antagonist, AM1476. The compound was orally administered at 1,10 and $30 \mathrm{mg} / \mathrm{kg}$ b.i.d. from day 21 , several days after the first clinical signs of cGvHD, to day 49. Dermal thickness, myofibroblast counts and collagen production were used to evaluate dermal fibrosis. Effects on pulmonary fibrosis were measured using hydroxyproline content, Sirius Red staining and Ashcroft score. Plasma was collected for PK analysis at different timepoints in each treatment group using sparse sampling, on the last day of the experiment. The tight-skin1 model was used to evaluate anti-fibrotic effects after therapeutic treatment. AM1476 was orally administered at $10 \mathrm{mg} / \mathrm{kg}$, b.i.d. from week 5 to week 10. Hypodermal thickening, myofibroblast counts and hydroxyproline content in skin biopsies were evaluated at the end of the treatment period. The number of phosphorylated Smad3 (pSmad3) positive cells was used to evaluate inhibition of TGF- $\beta$ signaling.

Results: The $5-\mathrm{HT}_{2 \mathrm{~B}}$ receptor antagonist $\mathrm{AM} 1476$, significantly reduced all measured dermal and pulmonary fibrosis readouts in the cGvHD model using an oral therapeutic treatment approach. Pharmacokinetic analysis of plasma samples supported $5-\mathrm{HT}_{2 \mathrm{~B}}$ receptor engagement. Therapeutic treatment of dermal fibrosis in the tight-skin model effectively and significantly reduced hypodermal thickening, number of myofibroblast and hydroxyproline content. The number of pSmad3 positive cells was significantly reduced in skin samples isolated from treated animals.

Conclusion: Inhibition of $5-\mathrm{HT}_{2 \mathrm{~B}}$ receptor activity resulted in pronounced anti-fibrotic effects in pulmonary and dermal fibrotic tissues. AM1476 was well tolerated without obvious signs of toxicity. Effects on pSmad3, reflecting TGF- $\beta$-inhibition, will be evaluated as a potential biomarker in upcoming clinical trials. The highly selective $5-\mathrm{HT}_{2 \mathrm{~B}}$ receptor antagonist AM1476 represents a promising drug candidate for treatment of fibrotic conditions and is currently in development for systemic sclerosis.

Disclosure of Interests: Christina Wenglén Employee of: Employed by AnaMar AB, Lund, Sweden, Helena Arozenius Employee of: AnaMar AB, Lund, Sweden, Lars Pettersson Employee of: AnaMar AB, Lund, Sweden, Gunilla Ekstrom Employee of: AnaMar AB, Lund, Sweden DOI: 10.1136/ annrheumdis-2019-eular.6808

\section{THU0354 MACHINE LEARNING CLASSIFICATION OF SKIN GENE EXPRESSION IDENTIFIES A SUBSET OF SYSTEMIC SCLEROSIS PATIENTS MOST LIKELY TO SHOW CLINICAL IMPROVEMENT IN RESPONSE TO ABATACEPT}

Jennifer Franks ${ }^{1}$, Bhaven Mehta ${ }^{1}$, Veronica Berrocal ${ }^{2}$, Yue Wang ${ }^{1}$, Tammara Wood ${ }^{1}$, Christopher Denton ${ }^{3}$, Robert Lafyatis ${ }^{4}$, David Fox ${ }^{2}$, Dinesh Khanna ${ }^{2}$, Michael Whitfield ${ }^{1} .{ }^{1}$ Geisel School of Medicine at Dartmouth, Hanover, United States of America; ${ }^{2}$ University of Michigan, Ann Arbor, United States of America; ${ }^{3}$ Royal Free Hospital, London, United Kingdom; ${ }^{4}$ University of Pittsburgh, Pittsburgh, United States of America

Background: A prior pilot study of abatacept in SSc with molecular gene expression data in skin (1) found that four of five patients who improved on abatacept, as determined by change in mRSS, were in the inflammatory intrinsic gene expression subset. Improvement was accompanied by a decrease in gene expression for immune pathways, including the CD28 and CTLA4 receptors - the target of abatacept. Here we tested our a priori hypothesis that the inflammatory subset shows a significant decline in mRSS during abatacept therapy.

Objectives: ASSET (Abatacept Systemic SclErosis Trial) is a Phase 2 study designed to assess the efficacy of subcutaneous abatacept to treat diffuse cutaneous systemic sclerosis. We performed RNA-sequencing (RNA-seq) analysis of skin biopsies and analyzed associations between intrinsic molecular subsets and clinical outcomes in the ASSET trial.

Methods: RNA-seq was performed on skin biopsies from 84 participants in the ASSET trial. A machine learning classifier was trained on independent cohorts and used to objectively classify patients into intrinsic gene expression subsets prior to study unblinding (2). Treatment differences in longitudinal outcomes were assessed using linear mixed effect models.

Results: 84 participants were assigned to intrinsic subset at baseline resulting in 33 inflammatory, 18 fibroproliferative, and 33 normal-like samples. Change in mRSS over 12 months was significantly different between the abatacept and placebo treatment arms for the inflammatory $(p<0.001)$ and normal-like $(p=0.03)$ subsets, but there was no difference in the fibroproliferative subset of patients $(p=0.47)$ (Figure 1). For FVC\% predicted, the fibroproliferative subset showed a numerical increase in FVC\% in the abatacept arm $(p=0.19)$ while all other groups showed decreases in FVC\%. All gene expression subsets showed decreases in HAQ-DI in the abatacept arm not observed in the placebo arm, with the most robust changes occurring in the inflammatory $(p=0.09)$ and normallike $(p=0.06)$ subsets.

Conclusion: There was a marked divergence of the trajectory for mRSS change for the inflammatory subset and no apparent impact for the fibroproliferative subset. In contrast, only the fibroproliferative subset showed a clinically meaningful FVC change for abatacept. For the broader functional measure of HAQ-DI, there were similar impacts of abatacept on all three intrinsic skin gene expression subsets but is numerically greatest for the normal-like and fibroproliferative subsets. Together these data show for the first time in a placebo-controlled trial that intrinsic skin gene expression subsets may predict differential response to a targeted biological therapy, and also that this may reflect impact on different facets of SSc pathogenesis in skin or lung. This suggests that stratification of cases according to intrinsic gene expression subsets may maximize the number of informative SSc cases in clinical trials, and potentially improve future clinical practice.
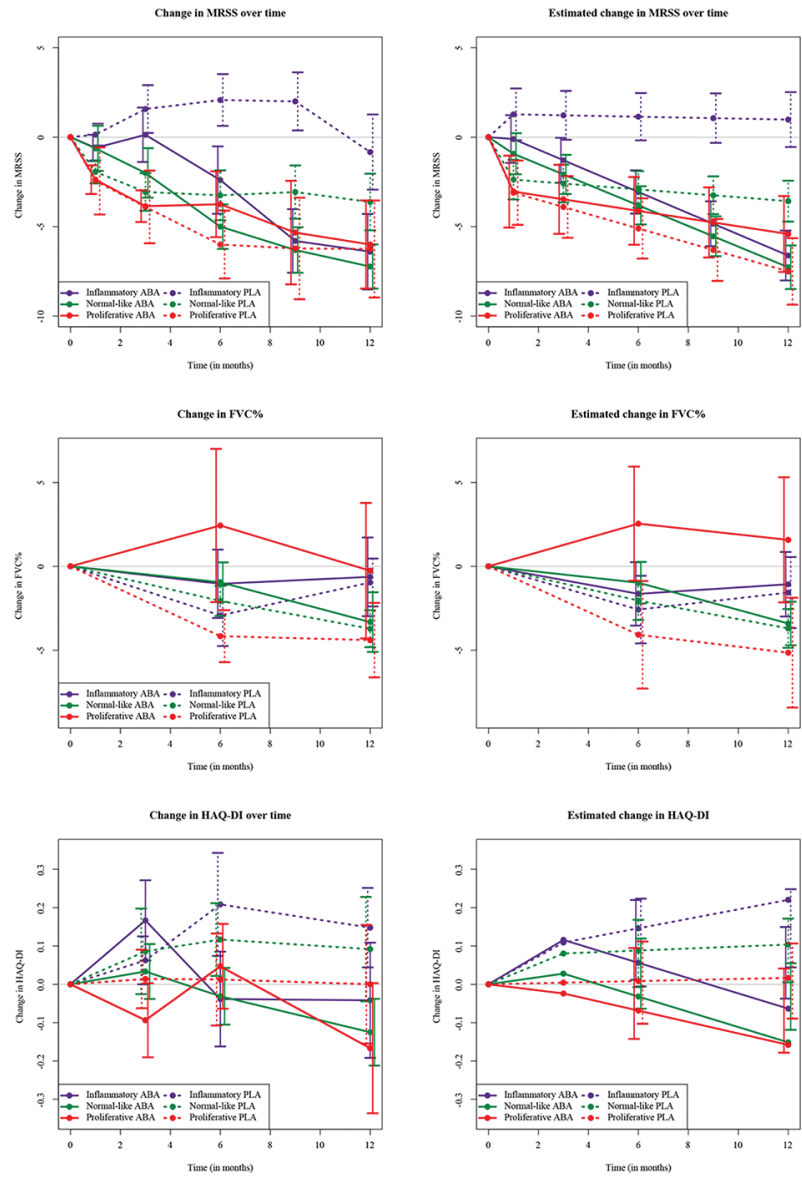

Abstract THU0354 - Figure 1

\section{REFERENCES}

[1] E. F. Chakravarty, et al., Gene expression changes reflect clinical response in a placebo-controlled randomized trial of abatacept in patients with diffuse cutaneous systemic sclerosis. Arthritis Res Ther 17, 159 (2015)

[2] J. M. Franks, et al., A Machine Learning Classifier for Assigning Patients with Systemic Sclerosis to Intrinsic Molecular Subsets., Arthritis Rheum. (2019) In Press.

Acknowledgement: This project was supported by NIH/NIAID Clinical ACE grant (5UM1Al110557-05) and an investigator-initiated grant by Bristol-Myers Squibb. Disclosure of Interests: Jennifer Franks: None declared, Bhaven Mehta: None declared, Veronica Berrocal: None declared, Yue Wang: None declared, Tammara Wood Consultant for: Celdara Medical LLC, Christopher Denton Grant/research support from: GlaxoSmithKline, Inventiva, CSF Behring, Consultant for: Roche-Genentech, Actelion, GlaxoSmithKline, 
Sanofi Aventis, Inventiva, CSL Behring, Boehringer Ingelheim, Bayer, Robert Lafyatis: None declared, David Fox: None declared, Dinesh Khanna Shareholder of: Eicos Sciences, Inc, Grant/research support from: Bayer, BMS, Pfizer, Horizon, Consultant for: Actelion Acceleron, Arena, Bayer, BI, BMS, CSL Behring, Corbus, Cytori, GSK, Genentech/Roche, Galapagos, Employee of: Elcos Sciences, Inc, Michael Whitfield Shareholder of: Scientific Founder, Celdara Medical LLC, Grant/research support from: Dr. Whitfield has received research contracts from Celdara Medical LLC and UCB Biopharma, Consultant for: Bristol Myers Squib, Celdara Medical, Corbus, and Boerhinger Ingelheim

DOI: 10.1136/annrheumdis-2019-eular.7661

\section{THU0355 IGURATIMOD MIGHT TREAT SCLERODERMA WITH INTERRUPTED EGR1/TGF-B LOOP}

Qingran Yan ${ }^{1}$, Lichong Shen ${ }^{1}$ Daniel Morales-Cano ${ }^{1}$ Xiaoxiang Chen ${ }^{1} .{ }^{1}$ Renji Hospital, Shanghai Jiaotong University, School of Medicine, Department of Rheumatology, Shanghai, China

Background: Systemic sclerosis (SSc) is an autoimmune disease characterized with multiple organ fibrosis. Previous studies showed transcription factor early growth response 1 (Egr1) overexpressed in the lesional skin of SSc patients, as well as Egr1 inducible genes ${ }^{1}$. Egr1 forms a positive feedback loop with master pro-fibrotic cytokine TGF $\beta$ and thus promotes fibrosis.

Objectives: To investigate the anti-fibrotic effect of a novel DMARD iguratimod in scleroderma models and patient skin grafts.

Methods: We used iguratimod to treat TGF $\beta$-stimulated human skin fibroblast, bleomycin induced mice, tight skin 1 (TSK-1) mice and SSc skin grafts. The bleomycin model contained pre-establish fibrosis and late onset treatment. The skin grafts came from three SSc patients and was planted into irradiated nude mice. Results: Iguratimod down-regulated egr1 expression in human skin fibroblast, with decreased collagen production and $\alpha$-SMA expression. Knocking down Egr1 in fibroblast could mimic these effects. Both oral and topical iguratimod could reduce dermal thickening and collagen deposition in bleomycin induced skin fibrosis. $\alpha$-SMA (+) myofibroblast counts, as well as Egr1 (+) and/or TGF $\beta$ (+) fibroblast counts in iguratimod treated groups were significantly less than the controls. Similarly, topical iguratimod ameliorated fibrosis with deduced dermal thickening in TSK-1 mice. Of note, 5-week iguratimod local injection remarkably reduced collagen content in skin grafts from three SSc patients. Staining of Egr1 and TGF $\beta$ in skin tissue were inhibited after iguratimod treatment simultaneously.

Conclusion: We found the potential of iguratimod to treat SSc, which was characterized as an Egr1 inhibitor. Further clinical investigation is needed to establish its safety and efficacy.

\section{REFERENCES}

[1] Bhattacharyya S, Sargent JL, Du P, et al. Egr-1 induces a profibrotic injury/repair gene program associated with systemic sclerosis. PloS one 2011;6(9):e23082 doi: 10.1371/journal.pone.0023082

[2] Chen SJ, Ning H, Ishida W, et al. The early-immediate gene EGR-1 is induced by transforming growth factor-beta and mediates stimulation of collagen gene expression. The Journal of biological chemistry 2006;281 (30):21183-97 doi: 10.1074/jbc.M603270200

[2] Wu M, Melichian DS, de la Garza M, et al. Essential roles for early growth response transcription factor Egr-1 in tissue fibrosis and wound healing. The American journal of pathology 2009;175(3):1041-55 doi: 10.2353/ ajpath.2009.

Acknowledgement: This work is supported by National Natural Science Foundation of China (81601401).

Disclosure of Interests: None declared

DOI: 10.1136/annrheumdis-2019-eular.2421

\section{THU0356 STAPHYLOCOCCUS AUREUS REGULATES FIBROBLAST FUNCTIONS - IMPLICATIONS FOR TISSUE REPAIR IN CHRONIC DIGITAL ULCERS IN SYSTEMIC SCLEROSIS}

Masaya Yokota ${ }^{1}$, Matthew Kassier ${ }^{1}$, Nicola Häffner ${ }^{2}$, Matthias Brunner ${ }^{1}$, Janine Schniering ${ }^{1}$, Oliver Distler ${ }^{1}$, Annelies S. Zinkernagel ${ }^{2}$, Britta Maurer ${ }^{1}$. ${ }^{1}$ Center of Experimental Rheumatology, Department of Rheumatology, University Hospital Zurich, Zurich, Switzerland; ${ }^{2}$ Department of Infectious Diseases and Hospital Epidemiology, University Hospital Zurich, University of Zurich, Zurich, Switzerland

Background: Chronic digital ulcers (DU) are a major complication in systemic sclerosis (SSc). Non-healing wounds are characterized by persistent inflammation, defective re-epithelialization and impaired matrix remodeling, and are often accompanied by bacterial colonization. In DU, the breach of the basement membrane exposes tissue and cell layers to commensa skin bacteria.

Objectives: To investigate whether interactions between commensal skin bacteria and dermal fibroblasts affect tissue repair mechanisms.

Methods: Dermal fibroblasts isolated from healthy controls $(\mathrm{HC})$ and patients with diffuse cutaneous SSc (dcSSc) ( $n=3$, each) were co-cultured with Staphylococcus aureus (SA) for $3 \mathrm{~h}$ at using a lower inoculum rather representing colonization $\left(1 \times 10^{5} \mathrm{CFU} / \mathrm{ml}\right)$ and a higher inoculum reflecting infection $\left(1 \times 10^{7} \mathrm{CFU} / \mathrm{ml}\right)$. Thereafter, fibroblasts were cultured with flucloxacillin-containing medium to kill extracellular and adherent bacteria. For mechanistic studies, a fibroblast cell line (BJ-5ta) was used. IL 6 , IL-8, pro-collagen $\mid \alpha$, and interferon (IFN)- $\beta$ proteins in culture supernatants were measured by ELISA. Gene expression was assessed by quantitative PCR. Contractility was analyzed by collagen gel contraction assay. Wound closure assay was performed on a confluent cell layer with a uniform cell-free gap, generated by a cell culture insert. Expression of alpha-smooth muscle actin ( $\alpha$-SMA) was evaluated by Western blotting, cell proliferation with a colorimetric assay. Cell death was ana lyzed by live cell real time assay. Bacterial invasion was assessed by immunofluorescence staining using confocal microscopy and CFU recovery. Gene knockdown was performed using siRNA.

Results: Exposure to $1 \times 10^{7} \mathrm{CFU} / \mathrm{ml}$ of $\mathrm{SA}$ influenced fibroblast function. In brief, exposure to SA increased the secretion of IL-6 and IL-8 in derma fibroblasts by $1571 / 455$-fold (HC/dcSSc; $p=0.08 /<0.05$ ) and by $325 / 1402$-fold ( $\mathrm{HC} / \mathrm{dcSSc}$; $\mathrm{p}<0.05$ each) respectively. In addition we observed upregulation of the expression of MMP1 by $8.2 / 5.7$-fold (HC/dcSSc; $p<0.05$ each) and MMP3 by $5.1 / 5.7$-fold $(\mathrm{HC} / \mathrm{dcSS}$; $\mathrm{p}=0.06 /<0.05)$. There was a trend towards decreased secretion of pro-collagen $1 \alpha$. TGF- $\beta$-induced $\alpha$-SMA expression was completely inhibited. Cell contraction was impaired by $22.0 / 31.6 \%$ (HC/dcSSc; $\mathrm{p}=0.08 /<0.05$ ), wound closure by $18.8 / 14.9 \%$ (HC/dcSSc; $p<0.05$ each), and proliferation by $30.5 / 44.3 \%$ (HC/dcSSc; $p=0.24 /<0.05)$. SA induced apoptosis and necrosis. SA invaded fibroblasts via endocytosis $(25-50 \%$ of inoculated live SA). IFN- $\beta$ secretion was induced 8.4/31.7-fold (HC/dcSSc; $p<0.05$ each). The genes of cytosolic dsDNA sensor molecules such as cyclic GMP-AMP synthase (cGAS) were upregulated 11.9/8.6-fold (HC/dcSSc; $\mathrm{p}<0.05$ each). TLR9, an endosomal DNA sensor, was constitutively expressed. Knockdown of STING or MyD88, downstream mediators of cGAS and TLR9 respectively, reduced the induction of IFN- $\beta$ (down to 68.2/66.2\%; $\mathrm{p}=0.20 / 0.16$ ), IL-6 (down to $57.2 / 55.2 \% ; \mathrm{p}<0.05$ each), and IL-8 (down to $45.3 / 36.0 \% ; \mathrm{p}<0.05$ each). Furthermore, inhibition of endocytosis with cytochalasin $D$ decreased the expression of IFN- $\beta$ (down to $24.6 \%$; $<<0.05$ ), IL-6 (down to $34.9 \%$; $p<0.05$ ), and IL-8 (down to 27.6\%; $\mathrm{p}<0.05$ ). In contrast, inhibition of endocytosis did not affect induction of apoptosis. This suggests that the invasion of fibroblasts by $\mathrm{SA}$ and the subsequent intracellular DNA sensing are crucial for the induction of these genes independently of apoptosis.

Conclusion: Invasion of dermal fibroblasts by SA with activation of the STING and TLR9-MyD88 pathways is a key element in the impairment of tissue repair responses.

\section{THU0357 VENTRICULAR-ARTERIAL COUPLING AS A PREDICTOR OF CARDIOVASCULAR EVENTS IN SYSTEMIC SCLEROSIS}

Elisabetta Zanatta ${ }^{1}$, Pamela Polito ${ }^{1}$, Giulia Famoso ${ }^{2}$, Franco Cozzi ${ }^{1}$, Andrea Doria ${ }^{1}$, Francesco Tona ${ }^{2} .{ }^{1}$ Rheumatology Unit, Department of MedicineDIMED, Padova, Italy; ${ }^{2}$ Department of Cardiac, Thoracic and Vascular Sciences, University of Padova, Padova, Italy

Background: Standard Transthoracic Echocardiography (TTE) parameters have shown a low sensitivity in the detection of primary miocardia involvement (PMI) in systemic sclerosis (SSc). Arterial-ventricular coupling (VAC) is calculated by TTE as the ratio between arterial elastance (Ea) and ventricular end-systolic elastance (Ees), which reflects left ventricle stifness. VAC is a central determinant of cardiovascular performance.

Objectives: We aimed to assess TTE-derived measures of cardiac mechanics and the prognostic role of VAC in SSc.

Methods: 75 patients affected by SSc without symptoms of cardiac involvement and 10 controls matched for sex and age were retrospectively evaluated. We considered the first TTE available after SSc diagnosis. Determinants of cardiac mechanics were calculated by TTE ${ }^{1}$ : end systolic elastance (Ees), arterial elastance (Ea), ventricular arterial cou pling $(\mathrm{VAC}=\mathrm{Ees} / \mathrm{Ea})$, end diastolic elastance (Eed). A value of VAC > 0.62 was considered indicative of altered VAC (i.e. uncoupling), whereas a lower value as normal (i.e. coupling). Finally, we considered the hospitalization for cardiovascular event during a mean follow-up of $10.8 \pm 1.8$ years. 